\title{
Candidate Defense Genes as Predictors of Quantitative Blast Resistance in Rice
}

\author{
Bin Liu, ${ }^{1,3,4}$ Shaohong Zhang, ${ }^{1}$ Xiaoyuan Zhu, ${ }^{2}$ Qiyun Yang, ${ }^{2}$ Shangzhong Wu, ${ }^{2}$ Mantong Mei, ${ }^{3}$ Ramil \\ Mauleon, ${ }^{4}$ Jan Leach, ${ }^{5}$ Tom Mew, ${ }^{4}$ and Hei Leung ${ }^{4}$ \\ ${ }^{1}$ Rice Research Institute and ${ }^{2}$ Plant Protection Research Institute, Guangdong Academy of Agricultural Sciences, \\ Guangzhou 510640, P. R. China; ${ }^{3}$ College of Life Sciences, South China Agricultural University, Guangzhou 510642, P. R. \\ China; ${ }^{4}$ Entomology and Plant Pathology Division, International Rice Research Institute, DAPO Box 7777, Manila, \\ Philippines, ${ }^{5}$ Department of Plant Pathology, Kansas State University, Manhattan 66506-5502, U.S.A.
}

Submitted 25 February 2004. Accepted 25 June 2004.

\begin{abstract}
Although quantitative trait loci (QTL) underpin many desirable agronomic traits, their incorporation into crop plants through marker-assisted selection is limited by the low predictive value of markers on phenotypic performance. Here we used candidate defense response (DR) genes to dissect quantitative resistance in rice using recombinant inbred (RI) and advanced backcross (BC) populations derived from a blast-resistant cultivar, Sanhuangzhan 2 (SHZ-2). Based on DNA profiles of DR genes, RI lines were clustered into two groups corresponding to level of resistance. Five DR genes, encoding putative oxalate oxidase, dehydrin, PR-1, chitinase, and 14-3-3 protein, accounted for 30.0, 23.0, 15.8, 6.7, and $5.5 \%$ of diseased leaf area (DLA) variation, respectively. Together, they accounted for $60.3 \%$ of the DLA variation and co-localized with resistance QTL identified by interval mapping. Average phenotypic contributions of oxalate oxidase, dehydrin, PR-1, chitinase, and 14-3-3 protein in BC lines were 26.1, 19.0, 18.0, 11.5, and $10.6 \%$, respectively, across environments. Advanced BC lines with four to five effective DR genes showed enhanced resistance under high disease pressure in field tests. Our results demonstrate that the use of natural variation in a few candidate genes can solve a long-standing problem in rice production and has the potential to address other problems involving complex traits.
\end{abstract}

Additional keywords: defense response gene, Magnaporthe grisea.

Availability of abundant molecular markers for genetic mapping has led to the rapid identification of quantitative trait loci (QTL) conditioning a variety of plant traits (Paterson et al. 1988; Stuber et al. 1992; Wang et al. 1994). However, applications of marker-assisted selection for improving quantitative traits in plant breeding are limited by a low predictive value of QTL markers for performance. This problem is caused by a variety of factors, including recombination between target traits and markers, low expression of certain genes, and strong genotype-environment interaction (Paterson et al. 1991; Young 1996). Genome sequencing projects have made it pos-

Corresponding author: Hei Leung; Telephone: 63-2-845-0563 (extension 2723); Fax: 63-2-854-0606; E-mail: H.Leung@ cgiar.org

* The $\boldsymbol{e}$-Xtra logo stands for "electronic extra" and indicates the HTML abstract available on-line contains four supplemental tables not included in the print edition. sible to use known candidate gene sequences as markers to associate target traits. Sequence information can be used to infer the function of a particular gene. If the gene confers the trait of interest, then the recombination problem associated with marker-assisted selection is alleviated. Regardless of marker type, a prerequisite for successful marker-assisted selection for QTL is knowledge of the predictive value of the markers and the robustness of the prediction in different environments. Although associations of candidate gene markers with QTL exist (Faris et al. 1999; Ramalingam et al. 2003), no critical analysis has been done to show the practical utility of candidate genes as predictors of QTL. Furthermore, few experimental data exist that allow the assignment of phenotypic values to markers such that breeding gain can be predicted through the accumulation of specific alleles.

Rice blast disease, caused by the fungus Magnaporthe grisea (Hebert) Barr, is a useful model to test the application of the candidate gene approach to enhance disease control by incorporating QTL. Rice blast occurs in most rice-growing regions of the world, and control of the disease depends largely on host resistance conferred by major disease resistance $(R)$ genes (Bonman et al. 1992). With rare exceptions, major gene resistance, which often shows race specificity, is not durable because of the high degree of pathogenic variation (race change) exhibited by the fungus (Bonman et al. 1992). Wang and associates (1994) demonstrated that durable resistance to blast observed in a traditional rice cultivar is controlled by a combination of major $R$ genes and QTL. Quantitative resistance, in general, exhibits no race specificity and is believed to be more durable than major gene resistance. Although a preferred strategy, selection for QTL is complicated by the masking effect of major $R$ genes. The low resolution of QTL mapping also precludes the effective use of markers for breeding. Furthermore, it is difficult to accumulate quantitative resistance without knowledge of the underlying genetic control (Michelmore 2003). Consequently, no cultivar with broadspectrum QTL-based resistance has been developed based on prediction of genetic markers.

Here, we use rice cv. Sanhuangzhan 2 (SHZ-2), which exhibits durable blast resistance, to test the hypothesis that genetic markers derived from candidate defense response (DR) genes are superior predictors of phenotypic performance. The DR genes used in this study are involved in the defense response process. They are recognized based on their increased expression during plant defense. The proteins encoded by these genes include i) structural proteins that are incorporated into the extracellular matrix and participate in the confinement of the 
pathogen, ii) enzymes of secondary metabolism, and iii) enzymes, which are implicated to be directly involved in the defense response. We first used a recombinant inbred (RI) mapping population to identify candidate DR genes linked to loci with large phenotypic effects. The utility of these markers for selecting quantitative resistance was validated using an advanced backcross (BC) population. By evaluating the genetic materials against diverse pathogen populations in multiple locations, we quantified the average phenotypic effects of the DR genes across environments. The empirical data demonstrate that selection for candidate genes can effectively accumulate QTL conferring broad-spectrum resistance and that specific alleles of DR genes are good predictors of quantitative resistance in multiple environments.

\section{RESULTS}

\section{SHZ-2 has both qualitative}

and quantitative resistance to blast.

We first evaluated the range of resistance of SHZ-2 by artificial inoculation with 344 blast isolates from eight provinces in China and 50 isolates representing 14 diverse genetic lineages in the Philippines (Chen et al. 1995). SHZ-2 expressed resistance to $96 \%$ of the isolates from China and $98 \%$ of the isolates from the Philippines, thus confirming the broad resistance of the cultivar. A molecular linkage map locating genes conferring qualitative resistance to blast was constructed using 48 restriction fragment length polymorphism (RFLP) markers, 26 simple-sequence repeat (SSR) markers, and 87 candidate DR gene markers. By analyzing the co-segregation between disease phenotypes and molecular markers in RI lines, three major $R$ genes conferring resistance to two isolates from Guangdong and one isolate from the Philippines were identified and tentatively designated as $P i-G D-1(\mathrm{t}), P i-G D-2(\mathrm{t})$, and $P i-G D-3(\mathrm{t})$ (Fig. 1). $P i-G D-1(\mathrm{t})$ was mapped on chromosome $8,3.3$ centimorgans $(\mathrm{cM})$ from a putative oxalate oxidase marker. $P i-G D-2(\mathrm{t})$ was mapped on chromosome $10,3.9 \mathrm{cM}$ from a nucleotide binding site leucine-rich repeater (NBSLRR) marker (r16), and Pi-GD-3(t) was mapped on chromosome $12,4.8 \mathrm{cM}$ from the SSR marker RM179.

To determine the level of quantitative resistance in SHZ-2, we evaluated resistance of the RI lines in both greenhouse and field (natural infestation) tests in China and the Philippines. Different disease reactions of the RI lines across the three field locations were observed, reflecting different blast pathogen population structures. The distribution of diseased leaf area (DLA) among RI lines in both greenhouse and field experiments was skewed toward resistance, presumably due to the presence of major $R$ genes. To remove the masking effect of major genes, we analyzed $101 \mathrm{RI}$ lines that did not carry markers closely linked to the major gene conferring resistance to isolate PO6-6 and showed susceptible reactions to isolate PO6-6 in inoculation experiments. Normal distribution patterns of DLA were observed among the $101 \mathrm{RI}$ lines (data not shown). A wide range of quantitative resistance was observed between the most resistant $10 \%$ (DLA less than $9.0 \%$ ) and least resistant $10 \%$ of the RI population (DLA greater than 35.6\%) under field and greenhouse assays. Pairwise analyses of DLA on RI lines from different experiments showed significant correlation in levels of resistance between tests $(r=0.79 * *$ for Guangdong greenhouse versus International Rice Research Institute [IRRI] greenhouse, $r=$ $0.72 * *$ for Guangdong greenhouse versus IRRI field, $r=0.89 * *$ for IRRI field versus IRRI greenhouse, and $r=0.70 * *$ for IRRI field versus Cavinti field). The high correlations in levels of resistance against diverse pathogen isolates under field conditions suggest that the quantitative resistance to blast derived from SHZ-2 is not race specific.

\section{Associations between DR genes and blast quantitative resistance.}

To determine the relationship between DR genes and blast QTL, 20 RI lines from each of the "tails" of the distribution curve, representing high and low DLA, were selected for analysis using 20 DR genes and 20 randomly selected SSR markers. Based on the DNA fingerprints derived from DR genes, the selected 40 RI lines were clustered into two groups corresponding to their levels of disease resistance (Fig. 2A). In contrast, the dendrogram produced from SSR analysis showed no relationship between the grouping of RI lines and the level of resistance (Fig. 2B).

Five heterologous DR gene probes were significantly associated with DLA in RI lines based on single-factor analysis of variance using all 101 RI lines (SAS general linear model [GLM] procedure), whereas other DR genes showed no significant association (Table 1). Oxalate oxidase exhibited the strongest effect, explaining $30.0 \%$ of DLA variation. PR-1 and
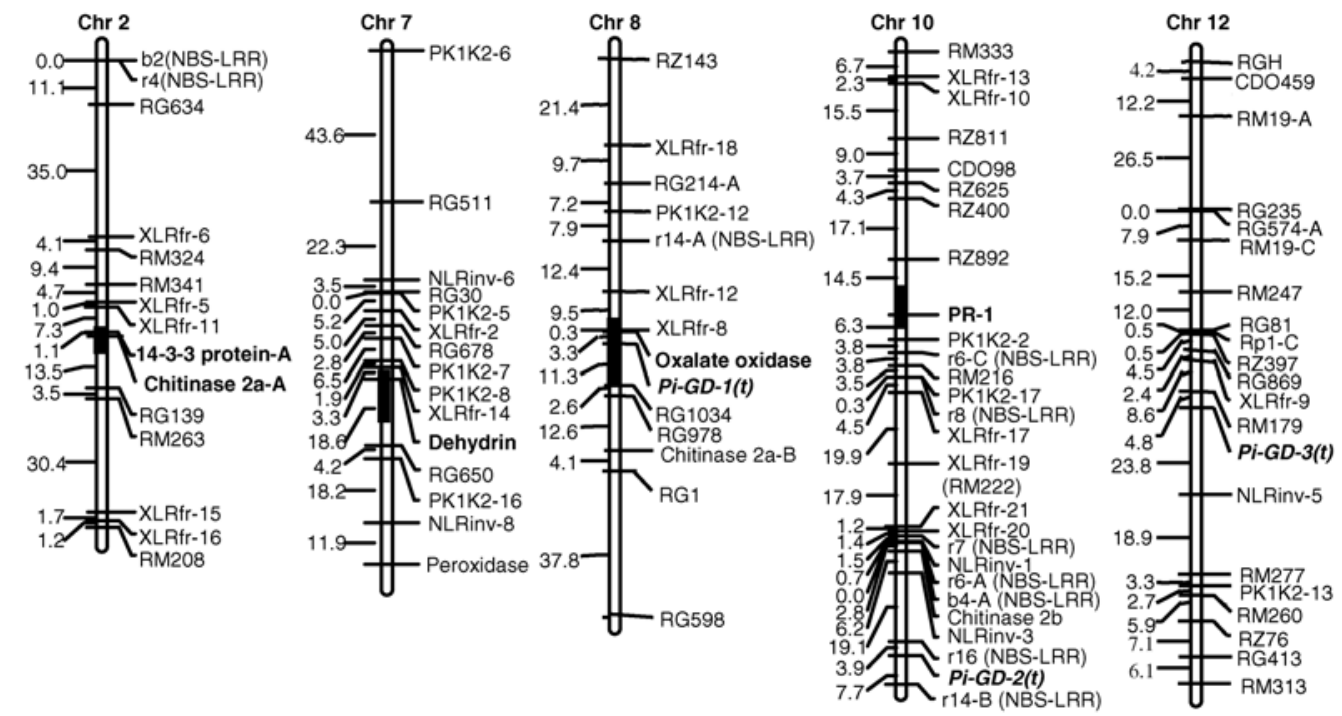

Fig. 1. Linkage map showing location of five defense genes: 14-3-3 protein, chitinase, dehydrin, oxalate oxidase, and PR-1; and three major resistance genes: $P i-G D-1(\mathrm{t}), P i-G D-2(\mathrm{t})$, and $P i-G D-3(\mathrm{t})$. Solid-filled areas on chromosomes represent the intervals of putative quantitative trait loci for diseased leaf area by interval mapping. 
dehydrin also had a strong effect, explaining 15.8 and $23.0 \%$ of DLA variation, respectively. Chitinase and the 14-3-3 protein had smaller effects on DLA variation. Together, these five DR markers or genes linked to them contributed $60.3 \%$ of the total DLA variation. Two-way interaction analysis using the GLM procedure in the SAS program indicated no significant interactions among the five effective alleles, suggesting that their actions are additive.

As a complementary analysis to determine the relationships between the DR genes and the blast-resistance QTL, we applied interval mapping using the entire marker dataset of 161 anchor and candidate gene markers (Mapmaker/QTL) (Lincoln et al. 1993). Three QTL with log of the likelihood ratio (LOD) scores $>3$ were identified (Fig. 1). One QTL on chromosome 8, defined by the interval of XLRfr-8-RG1034 (LOD = 7.1), explained $30.8 \%$ of DLA variation. The second QTL was on chromosome 7, defined by the interval of dehydrin-RG650 ( $\mathrm{LOD}=5.3$ ), and it accounted for $21.8 \%$ of DLA variation. The third QTL, located on chromosome 10, was defined by the interval of RZ892-PK1K2-2 (LOD = 3.8), and it explained $15.8 \%$ of DLA variation. The three DR genes with strong effects on the reduction of DLA (oxalate oxidase, dehydrin, and PR-1) were located within the intervals of the three top QTL identified by interval mapping. The other two DR genes, chitinase and 14-3-3 protein, are located in the QTL region on chromosome 2 but with a lower LOD score of 1.6. Thus, based on GLM-SAS and interval mapping analyses, we identified the same DR genes as the largest contributors to disease reduction in the progeny derived from the SHZ- $2 \times$ LTH cross. All disease-reducing QTL were from SHZ-2.

\section{Locating the candidate genes \\ with QTL effect on the rice genome physical map.}

Four of the five candidate genes were aligned against the International Rice Genome Sequencing Project (IRGSP) physical map of rice. Significant BLAST scores $\left(E<10^{-13}\right)$ demonstrated that four of the five candidate genes localized to the same chromosome where these genes were genetically mapped. Other significant scores occurred in different chromosomes, as expected for loci belonging to multigene families. The IRGSP physical map location confirms the genetic linkage of chitinase and 14-3-3 protein (1.1 cM apart on chromosome 2). Blast searches identified these two candidate genes in adjacent contigs (OJ1058_F07 and OJ1124_H01) that span approximately $1 \mathrm{Mb}$ on chromosome 2. Interestingly, the candidate gene oxalate oxidase, which was genetically mapped on chromosome 8 , had significant BLAST scores within a $52-\mathrm{kb}$ region in chromosome 8 that is annotated to contain nine homologous copies of germin-like protein (GLP) (i.e., oxalate oxidase) genes. A similar GLPgene rich region was reported on chromosome 4 of barley (Druka et al. 2002). PR-1 has sequence matches on two contigs, $36 \mathrm{cM}$ apart, on the physical map of chromosome 10. Thus, using defense genes from maize and barley, rice homologs associated with quantitative resistance can be identified readily in the rice genome database.

Sequences related to Esi18.5, a putative dehydrin, were detected on chromosome 7, where it was genetically mapped ( $E$ value $=8.5)$. However, the low alignment score indicates considerable divergence of this gene sequence from Lophopyrum elongatum from its rice orthologs and is consistent with the weak hybridization bands observed in RFLP analysis.

\section{Phenotypic contribution}

of defense response genes in diverse environments.

To quantify the value of the candidate genes as markers in a breeding program, we estimated the reduction in disease attributed to specific alleles of candidate genes across diverse envi-

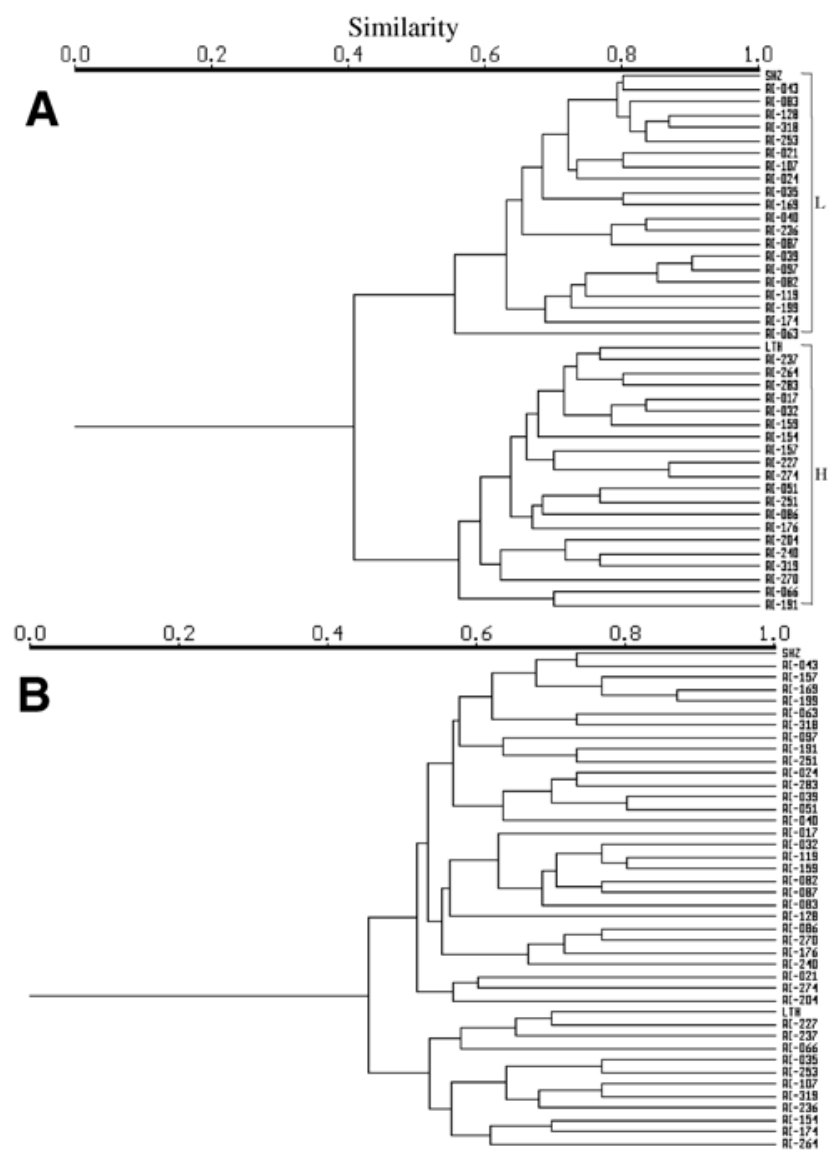

Fig. 2. Clustering of genotypes expressing high $(\mathrm{H})$ and low $(\mathrm{L})$ levels of blast resistance in the recombinant inbred lines using DNA fingerprints produced by candidate defense response gene and simple-sequence repeat (SSR) markers. A, Dendrogram derived from candidate gene fingerprints of 40 lines from the two tails of the normal distribution curve, showing strong groupings of resistant (L) and susceptible lines $(\mathrm{H})$. B, No grouping is evident when the dendrogram is produced using SSR fingerprints.

Table 1. Association between effective defense response genes and blast quantitative resistance in recombinant inbred (RI) lines evaluated under greenhouse and field conditions ${ }^{\mathrm{a}}$

\begin{tabular}{|c|c|c|c|c|c|c|}
\hline Defense response gene & Chromosome & IRRI-GH & GD-GH & GD-BN & IRRI-BN & Ca-BN \\
\hline Chitinase & 2 & $6.7 * *$ & $0.4 \mathrm{~ns}$ & $6.7^{*}$ & $5.0 *$ & $5.0 *$ \\
\hline $14-3-3$ protein & 2 & $5.5^{*}$ & $0.4 \mathrm{~ns}$ & $7.1 *$ & $5.0^{*}$ & $4.0^{*}$ \\
\hline Dehydrin & 7 & $23.0 * * * *$ & $21.8^{* * * * *}$ & $14.9 * * * *$ & $25.6^{* * * * *}$ & $16.7 * * * *$ \\
\hline Oxalate oxidase & 8 & $30.0 * * * *$ & $25.8 * * * *$ & $41.2 * * * *$ & $12.1 * * * *$ & $27.0^{* * * * *}$ \\
\hline PR-1 & 10 & $15.8 * * * *$ & $23.2 * * * *$ & $13.8 * * * *$ & $10.7 * * * *$ & $16.9 * * * *$ \\
\hline
\end{tabular}

a Experiments were conducted in the greenhouses of Guangdong, China (GD-GH) and the International Rice Research Institute (IRRI) in the Philippines (IRRI-GH); and the blast nurseries of Guangdong, China (GD-BN) and IRRI and Cavinti in the Philippines (IRRI-BN and Ca-BN). Value is the percent phenotypic contribution of a specific allele to diseased leaf area variation in RI lines at a given location. Level of significance: *, $* *$, and $* * * *$ indicate $P<$ $0.05,0.01$, and 0.0001 , respectively; ns $=$ no significant association at $P<0.05$. 
ronments. Using disease scores of RI lines in different assays (greenhouse and field), we compared the RI lines carrying donor alleles from SHZ-2 with those carrying the alternate alleles. RI lines with SHZ-2 alleles at oxalate oxidase, dehydrin, and PR-1 showed significantly lower DLA ( $t$ test, $P \leq 0.05)$ in all test locations (Table 2). The SHZ-2 alleles of these three DR genes reduced DLA by 14.6 to $30.8 \%$, with oxalate oxidase showing the strongest effect. The SHZ-2 alleles of chitinase and 14-3-3 protein reduced DLA by 8.6 and $12.4 \%$, respectively, and the effects were significant in some but not all assay conditions. In these experiments, the DLA of RI lines decreased with an increasing number of effective alleles (Fig. 3 ), indicating the cumulative effect of the DR genes on quantitative resistance. The RI lines were exposed to diverse pathogens under the different assay conditions; therefore, the effects of these genes are not race specific.

\section{Validating the contribution}

\section{of candidate genes in a different genetic background.}

To generalize the usefulness of the candidate gene markers, we evaluated the effects of the same alleles in a different genetic background. SHZ-2 was backcrossed to TXZ-13, a high-quality but blast-susceptible commercial cultivar, to produce a $\mathrm{BC}_{3} \mathrm{~F}_{3}$ population and quantitative resistance was evaluated by greenhouse inoculation and field tests. The presence or absence of the five effective DR genes was determined by molecular analysis of the $\mathrm{BC}_{3} \mathrm{~F}_{3}$ lines. Single-factor analysis of variance detected associations between the five DR genes contributing to a reduction in disease in the $\mathrm{BC}_{3} \mathrm{~F}_{3}$ lines under arti-

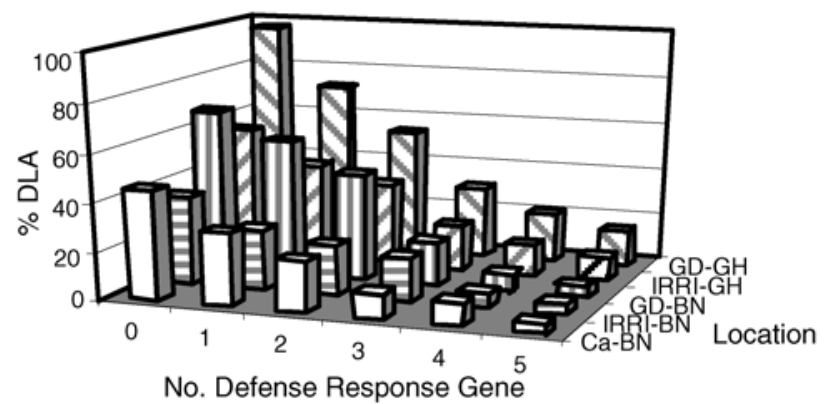

Fig. 3. Positive correlation between the level of resistance and the number of defense response genes in the recombinant inbred (RI) lines tested under greenhouse and field conditions. Means of diseased leaf area were calculated from RI lines carrying one, two, three, four, or five defense response genes in any possible combination. Locations refer to screening site at Cavinti (Ca-BN), blast nursery at International Rice Research Institute farm (IRRI-BN), screening site in Guangdong (GD-BN), greenhouse test at IRRI (IRRI-GH), and greenhouse test at Guangdong (GD-GH). ficial infection. As observed in the RI lines, oxalate oxidase exhibited the strongest effect on DLA reduction in $\mathrm{BC}_{3} \mathrm{~F}_{3}$ lines, with dehydrin and PR-1 also showing strong effects.

The cumulative effect of combining the five DR genes on the quantitative resistance was determined by evaluating all $\mathrm{BC}_{3} \mathrm{~F}_{3}$ lines in field tests. The genetic similarity of the top-performing lines was confirmed using SSR fingerprinting (50 polymorphic SSR markers from 12 chromosomes) (Fig. 4A). The selected resistant $\mathrm{BC}_{3} \mathrm{~F}_{3}$ lines were $>85 \%$ similar to the recurrent parent TXZ-13, as expected in $\mathrm{BC}_{3}$ population, confirming that these lines were products of backcrossing. $\mathrm{BC}_{3} \mathrm{~F}_{3}$ lines with three or more DR genes showed higher levels of blast quantitative resistance than the recurrent parent TXZ-13 in greenhouse and field tests conducted in China and the Philippines (Table 3). As observed in the RI lines, a significant correlation was found between DLA and the number of DR genes in individual $\mathrm{BC}_{3} \mathrm{~F}_{3}$ lines in the field tests (data not shown). Panicle blast was evaluated in two $\mathrm{BC}$ lines $(\mathrm{BC} 10$ and $\mathrm{BC} 116)$ in replicated trials over six cropping seasons in Guangdong. A significantly lower occurrence of panicle blast was observed in BC10
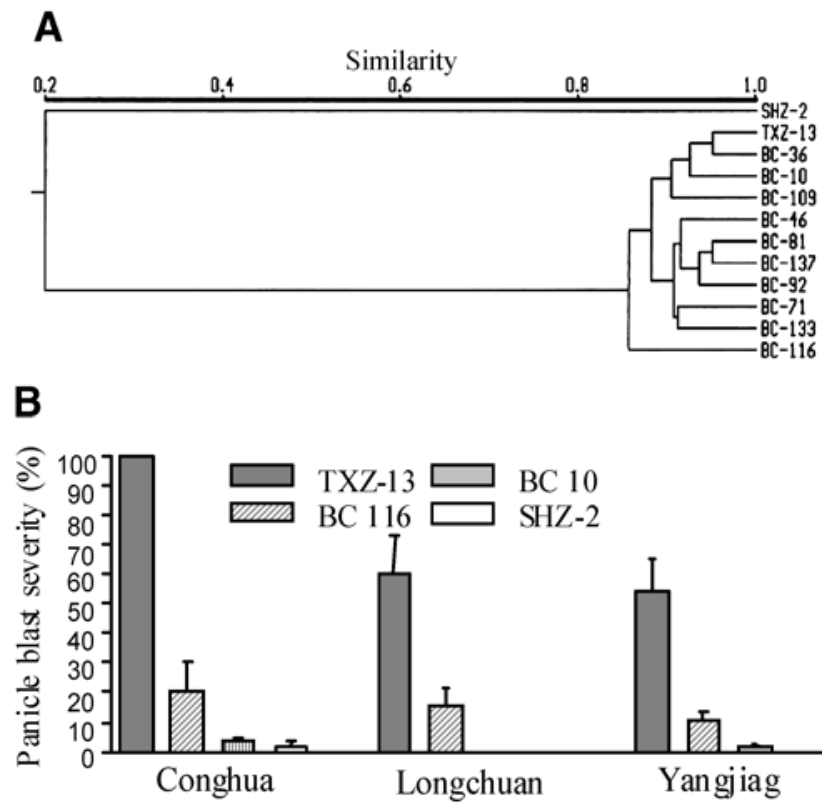

Fig. 4. Backcross lines with high level of blast resistance and the recurrent parent TXZ-13. A, Dendrogram produced by 50 simple-sequence repeat markers showing $>85 \%$ similarity between backcross lines and TXZ-13. B, Comparison of panicle blast severity among backcross lines (BC116 and BC10), the donor SHZ-2, and the recurrent parent TXZ-13 at three locations (Conghua, Longchuan, and Yangjiang) in Guangdong, China.

Table 2. Phenotypic effects of the five defense response genes in recombinant inbred (RI) lines at multiple locations ${ }^{\mathrm{a}}$

\begin{tabular}{|c|c|c|c|c|c|c|c|c|c|}
\hline \multirow[b]{2}{*}{ Defense gene } & \multirow[b]{2}{*}{ Allele } & \multirow[b]{2}{*}{ No. of lines } & \multicolumn{5}{|c|}{ DLA (\%) under five assay conditions } & \multirow[b]{2}{*}{ Avg. DLA (\%) } & \multirow[b]{2}{*}{ Reduction $(\%)^{\mathrm{b}}$} \\
\hline & & & IRRI-GH & GD-GH & IRRI-BN & $\mathbf{C a}-\mathbf{B N}$ & GD-BN & & \\
\hline \multirow[t]{2}{*}{ Oxalate oxidase } & - & 36 & $45.0 \pm 4.1$ & $74.5 \pm 7.0$ & $28.5 \pm 2.5$ & $47.7 \pm 4.8$ & $51.4 \pm 7.5$ & 49.4 & 30.8 \\
\hline & + & 65 & $19.5 \pm 3.6^{* * *}$ & $34.6 \pm 1.5^{* *}$ & $10.5 \pm 1.1 * * *$ & $16.7 \pm 1.3^{* * *}$ & $11.7 \pm 1.9 * * *$ & 18.6 & $\ldots$ \\
\hline \multirow[t]{2}{*}{ 14-3-3 protein } & - & 48 & $32.3 \pm 2.8$ & $52.8 \pm 5.0$ & $25.1 \pm 1.7$ & $28.7 \pm 3.0$ & $28.1 \pm 4.1$ & 33.4 & 12.4 \\
\hline & + & 53 & $21.1 \pm 2.1 *$ & $40.5 \pm 4.0$ & $10.6 \pm 1.0^{*}$ & $19.4 \pm 2.3$ & $13.5 \pm 2.5^{*}$ & 21.0 & $\ldots$ \\
\hline \multirow[t]{2}{*}{ Chitinase } & - & 47 & $31.3 \pm 2.8$ & $46.4 \pm 4.6$ & $24.8 \pm 1.8$ & $25.2 \pm 2.9$ & $25.8 \pm 4.1$ & 29.1 & 8.6 \\
\hline & + & 54 & $20.5 \pm 2.0^{*}$ & $41.3 \pm 3.8$ & $10.4 \pm 1.0^{*}$ & $17.2 \pm 2.2$ & $13.0 \pm 2.4^{*}$ & 20.5 & $\ldots$ \\
\hline \multirow[t]{2}{*}{ Dehydrin } & - & 45 & $36.9 \pm 3.4$ & $63.8 \pm 5.7$ & $29.3 \pm 2.4$ & $34.9 \pm 3.7$ & $36.9 \pm 5.1$ & 40.4 & 21.8 \\
\hline & + & 56 & $18.8 \pm 1.7 * *$ & $33.4 \pm 3.1 *$ & $9.2 \pm .0 .7 * * *$ & $19.2 \pm 2.1 *$ & $12.5 \pm 2.3 *$ & 18.6 & $\ldots$ \\
\hline \multirow[t]{2}{*}{ PR-1 } & - & 49 & $31.5 \pm 2.6$ & $47.5 \pm 4.3$ & $17.0 \pm 1.8$ & $28.7 \pm 2.6$ & $29.4 \pm 3.8$ & 30.8 & 14.6 \\
\hline & + & 52 & $17.7 \pm 1.8 *$ & $28.8 \pm 3.2 * *$ & $9.3 \pm 1.2 *$ & $13.1 \pm 2.3^{*}$ & $12.1 \pm 2.8 *$ & 16.2 & $\ldots$ \\
\hline
\end{tabular}

${ }^{\text {a }}$ The 101 RI lines were divided into two groups based on presence (+) or absence ( - ) of the effective allele. Each value represents the mean \pm standard error of the mean of diseased leaf area (DLA) of RI lines in one group at one location; *, **, and $* * *$ indicate $P<0.05,0.01,0.001$, respectively.

${ }^{\mathrm{b}}$ Average reduction in disease. Difference between the average DLA of $(+)$ and $(-)$ lines. 
and $\mathrm{BC} 116$ compared with the recurrent parent TXZ-13 in all three locations (Fig. 4B). Although both BC10 and BC116 carried the same five specific alleles from SHZ-2, the level of panicle blast resistance in $\mathrm{BC} 10$ was higher than that in BC116, suggesting that unidentified loci from SHZ-2 could have contributed to the difference in quantitative resistance. The data together indicate that the selected advanced BC lines carrying specific DR alleles have enhanced resistance against neck blast, the most damaging phase of the disease.

\section{DISCUSSION}

In rice, quantitative, non-race-specific resistance against blast disease is a desirable yet elusive trait because it is difficult to accumulate quantitative resistance without knowledge of the underlying genetic control. In this study, we used two independently derived genetic populations to show that selection for candidate DR gene markers is effective in accumulating a high level of quantitative resistance. We demonstrated that the level of resistance is proportional to the number of candidate gene alleles accumulated in advanced breeding lines and that the resulting resistance is non-race specific.

We hypothesized that the alleles of multiple DR genes in SHZ-2 collectively contributed to the high level of field resistance, and predicted that progeny allelic states in DR genes, but not at other loci, would be correlated with levels of resistance. Cluster analysis using candidate gene and SSR markers supports this hypothesis. RI lines that express a higher level of quantitative resistance (top 10\%) have multiloci haplotypes resembling SHZ-2. SSR markers did not reveal grouping of the lines according to resistance phenotype. The strong associations between candidate gene haplotypes and phenotypic performance suggest that the level of resistance of individual lines can be predicted according to their position on the dendrogram. Although the candidate gene profiles reflect resistance, only a few of the DR genes individually showed significant effects on disease reduction. Thus, candidate gene profiling can be used to reveal the collective contribution of alleles with minor effects that otherwise are difficult to exploit. Generalization of this approach will require experimentation with traits for which knowledge of candidate genes is available.

Consistent effects of five SHZ-2 alleles were observed in the $\mathrm{RI}$ and BC populations. In both genetic backgrounds, the ranking of the phenotypic contributions of these five genes is similar, with oxalate oxidase having the highest effect. Each of the five candidate genes was reported to be induced by stress or predicted to function in disease resistance. For example,
Esi18.5, a putative dehydrin, originally was isolated as an early-salt-stress-induced gene and was associated with salt tolerance in L. elongatum (Galvez et al. 1993). Dehydrins are induced under drought, low temperature, freezing, salinity, or abscisic acid application (Close 1996). However, similarity to a rice gene in the region on chromosome 7 could not be confirmed by alignment to the rice genome; therefore, a role for this gene in defense, if any, remains to be determined. Chitinase, which hydrolyzes the polymer chitin, a substrate found in fungi and insects, was associated with enhanced blast resistance in rice (Nishizawa et al. 1999).

Oxalate oxidase (also classified as germin-like oxalate oxidase) is predicted to act both directly and indirectly in plant defense responses. The enzyme converts oxalate into $\mathrm{CO}_{2}$ and $\mathrm{H}_{2} \mathrm{O}_{2}$ and releases free $\mathrm{Ca}^{2+}$ from Ca-oxalate. Expression of wheat germin oxalate oxidase in transgenic poplar leaves is thought to detoxify oxalic acid produced by the pathogen Septoria musiva, thereby conferring resistance to the fungus $(\mathrm{Hu}$ et al. 2003; Liang et al. 2001). Both $\mathrm{Ca}^{2+}$ and $\mathrm{H}_{2} \mathrm{O}_{2}$ are involved in signaling for plant defense (Levine et al. 1996; Price et al. 2002). In addition, $\mathrm{H}_{2} \mathrm{O}_{2}$ could be directly toxic to microbes (Grant and Loake 2000) or could contribute to the structural reinforcement of plant cell walls (Schweizer et al. 2000; Vallelian-Bindschedler et al. 1998). The multiple roles of oxalate oxidase may account for the high phenotypic effect conferred by the gene in multiple tests.

The 14-3-3 proteins are a class of signal transduction proteins that interact physically with their client protein when the client is specifically phosphorylated (Sehnke et al. 2002). This phosphorylation-dependent binding to the client is an important step in signal transduction leading to a wide array of biological functions, including response to stress (Sehnke et al. 2002). Clients of the plant 14-3-3 proteins include the plasma membrane $\mathrm{H}^{+}$-ATPase, which regulates turgor pressure through ion and water transport, and other ion-transport proteins (Boer 2002). Some of the earliest detectable signaling events in plant defense responses include transmembrane ion fluxes (Lamb and Dixon 1997); therefore, a role for the 14-3-3 proteins in plant defense responses can be speculated. A 14-33 protein gene is associated with a QTL for stem rust resistance in wheat (Faris et al. 1999). Although it is still unclear how PR-1, a pathogenesis-related protein, acts in plant defense, its expression frequently is associated with disease resistance in infected plants (Ward et al. 1991). Interestingly, both the PR-1 and 14-3-3 protein genes were identified in a genomewide search for genes involved in stress responses (Cooper et al. 2003).

Table 3. Composition of defense response genes in selected $\mathrm{BC}_{3} \mathrm{~F}_{3}$ lines derived from SHZ-2 or TXZ-13 and their levels of disease resistance in greenhouse and field tests ${ }^{\mathrm{a}}$

\begin{tabular}{|c|c|c|c|c|c|c|c|c|c|c|}
\hline \multirow[b]{2}{*}{ Cultivar or line } & \multirow[b]{2}{*}{ Chitinase } & \multirow[b]{2}{*}{ 14-3-3 protein } & \multirow[b]{2}{*}{ Dehydrin } & \multirow[b]{2}{*}{ Oxalate oxidase } & \multirow[b]{2}{*}{ PR-1 } & \multicolumn{5}{|c|}{ Average disease leaf area $(\%)^{b}$} \\
\hline & & & & & & GD-GH & GD-BN & IRRI-GH & IRRI-BN & Ca-BN \\
\hline SHZ-2 & + & + & + & + & + & 0 & 1.0 & 0 & 0 & 0 \\
\hline BC-10 & + & + & + & + & + & 9.6 & 4.5 & 0 & 0 & 0 \\
\hline BC-109 & - & + & + & + & + & 1.9 & 8.0 & 8.3 & 5.3 & 2.0 \\
\hline BC-116 & + & + & + & + & + & 2.4 & 8.0 & 7.7 & 5.6 & 1.1 \\
\hline BC-133 & + & + & + & + & + & 2.6 & 10.0 & 6.0 & 1.6 & 0.8 \\
\hline BC-137 & + & + & + & + & + & 4.7 & 10.0 & 10.2 & 7.5 & 2.4 \\
\hline BC-81 & + & + & + & + & + & 10.7 & 8.0 & 2.4 & 0.9 & 1.0 \\
\hline BC-36 & + & + & + & + & - & 5.0 & 5.0 & 15.9 & 2.3 & 1.6 \\
\hline BC-71 & + & + & - & + & + & 4.4 & 4.0 & 10.5 & 1.9 & 3.1 \\
\hline BC-92 & - & - & + & + & + & 8.8 & 6.0 & 13.2 & 2.3 & 1.8 \\
\hline BC-46 & + & + & + & - & + & 11.5 & 12.0 & 13.3 & 5.4 & 1.5 \\
\hline TXZ-13 & - & - & - & - & - & 29.3 & 16.0 & 34.7 & 45.3 & 12.6 \\
\hline
\end{tabular}

${ }^{a}$ Presence $(+)$ or absence $(-)$ of SHZ-2 alleles.

${ }^{\mathrm{b}}$ Experiments were conducted in the greenhouses of Guangdong, China (GD-GH) and the International Rice Research Institute (IRRI) in the Philippines (IRRI-GH); and the blast nurseries of Guangdong, China (GD-BN) and IRRI and Cavinti in the Philippines (IRRI-BN and Ca-BN). 
The random assortment of genetic background in the RI lines enabled us to estimate the allelic effects by comparing lines with and without the alleles under different assay conditions. Enhanced resistance ranged from $8.6 \%$ (chitinase) to as high as a $30.8 \%$ (oxalate oxidase) reduction in DLA. Several reports suggest that engineered expression of specific chitinases, either alone or in combination with other genes, enhances resistance in dicots (Jach et al. 1995; Shah 1997). Schweizer and associates (2000) showed that transient expression of germin in epidermal cells of wheat reduces penetration of the powdery mildew fungus by 20 to $65 \%$. Although direct comparisons of our results with these studies are not possible due to differences in assays and gene expression levels, the disease reduction achieved in our field studies by accumulation of natural alleles of the five DR genes is encouraging. The level of resistance achieved in the $\mathrm{BC}$ lines can be predicted by the number of SHZ-2 alleles at the five candidate DR gene loci (Table 3).

Although our results show that the chromosomal regions harboring the candidate genes confer non-race-specific resistance, we have not directly demonstrated causality between candidate genes and quantitative resistance (Michelmore 2003). There may be other factors contributing to quantitative resistance. First, functional genes not tagged by polymorphic markers are not detected. Second, within each chromosomal region harboring the candidate gene, there may be other DR genes. For example, there were 22 genes in the $126-\mathrm{kb}$ region containing the oxalate oxidase locus on chromosome 8 , many of which are predicted to encode hypothetical proteins of unknown function. Whether these adjacent genes contribute to enhanced resistance is not clear. We are developing near-isogenic lines to narrow the region of introgressed DNA so that the effects of the target genes can be assayed with minimal influence from other introgressed DNA segments. We also are examining the effects of individual genes by identifying knockout mutants.

In conclusion, we demonstrated the use of the candidate gene approach for effective selection of QTL for disease resistance. Whereas conventional QTL mapping starts with defining chromosomal intervals that would carry the candidate genes, the candidate gene approach begins with a target sequence, hence pinpointing the chromosomal regions for selection and increasing the predictive value of the markers. We associated specific alleles of candidate DR genes with quantitative values of blast resistance and showed that these five DR genes can provide non-race-specific resistance in multiple environments. Thus, it is possible to accumulate relatively few genes to achieve an adequate level of quantitative resistance to blast. This supports the use of natural variation in candidate genes to improve complex traits that seemingly are controlled by many genes.

\section{MATERIALS AND METHODS}

\section{Plant materials.}

SHZ-2 was crossed with Lijiangxin-tuan-heigu (LTH), a blast-susceptible japonica cultivar, and advanced from $\mathrm{F}_{2}$ to $\mathrm{F}_{8}$ by single seed descent; the 215 resulting RI lines were used for gene mapping. Blast-resistance QTL were transferred into the popular cv. Texianzhan-13 (TXZ-13), which is high yielding and has good quality but is susceptible to blast, and was achieved by backcrossing SHZ-2 to TXZ-13. BC lines phenotypically similar to the recurrent parent in each generation were crossed to TXZ-13 and selfed to produce the $\mathrm{BC}_{3} \mathrm{~F}_{3}$. Lines with poor agronomic traits were removed, leaving 144 $\mathrm{BC}_{3} \mathrm{~F}_{3}$ lines for molecular analysis and resistance evaluation.

\section{Evaluation of blast quantitative resistance.}

To evaluate quantitative resistance, two blast isolates that exhibit virulence to many rice genotypes, GDV-1 (Guangdong, China) and PO6-6 (Philippines), were used in greenhouse inoculation. A randomized complete block design with three replicates was used, with 15 plants/line/replicate inoculated and scored as described (Wang et al. 1994). Field tests were conducted in the blast nursery at Conghua, Guangdong, China; and blast nurseries at IRRI, Los Baños, Laguna, and at Cavinti, the Philippines in 2000 and 2001. Field studies used a randomized complete block design with three replicates with 50 plants/line planted in 16-by-1.2-m nursery plots. Three rows of cultivars consisting of diverse susceptible genotypes were planted around the plots to enhance natural infection. DLA was assessed at 2 weeks after sowing using the method described by Notteghem and associates (1981). Panicle blast was evaluated in advanced BC lines at three locations in Guangdong using a randomized complete block design with three replicates $\left(10 \times 1.3 \mathrm{~m}^{2}\right.$ per plot $)$. Incidence of panicle blast was measured based on infection on the node and spikelets per panicle by scoring 200 panicles per plot.

\section{Molecular marker analysis.}

Two categories of DNA markers were used: i) RFLP (Cornell University, Ithaca, NY, U.S.A.) and SSR markers (Life Technologies, Inc., Gaithersburg, MD, U.S.A.) with known positions on genetic maps (Causse et al. 1994; Temnykh et al. 2000) and ii) candidate gene markers, which collectively are called defense response genes and consist of resistance gene analogs (RGAs), NBS-LRR probes, and predicted DR genes (Ramalingam et al. 2003). Four RGA primers (Ptokin1/Ptokin2, NLRR-for/NLRR-rev, NLRR-inv1/NLRR-inv2, and XLRRfor/XLRR-rev) were used (Ramalingam et al. 2003). DNA blot analysis was conducted using the enhanced chemiluminescence nucleic acid labeling and detection system (Amersham, Chalfont, U.K.). SSR and RGA analyses were as described (Ramalingam et al. 2003; Temnykh et al. 2000).

\section{Cluster analysis.}

In all, 20 DR gene markers and 20 random SSR markers were used to analyze RI lines with extreme DLA. For each marker, DNA band data were converted into binary data $(1=$ band presence, $0=$ band absence). Similarity matrices were calculated using the Windist program (Yap and Nelson 1996) with simple matching coefficient. Dendrograms indicating the genetic relationship among the selected RI lines were constructed using the NTSYS-pc program, version 1.80 (Rohlf 1993).

\section{Mapping of markers and QTL.}

RFLP and SSR markers were used as anchor markers to locate candidate DR genes and construct a linkage map using 215 RI lines. Markers that did not deviate significantly from the expected 1:1 segregation $(P<0.05)$ in the RI lines were used to construct a linkage map using Mapmaker/Exp. 3.0 (Lincoln et al. 1993). Single-factor analysis of variance (GLM procedure in SAS software) (SAS Institute 1989) was used to identify candidate genes significantly associated with blast quantitative resistance. The relationships between the effective DR genes and blast QTL were determined by interval mapping using Mapmaker/QTL (Lincoln et al. 1993). LOD values $>3.0$ were interpreted as a QTL.

\section{ACKNOWLEDGMENTS}

This research was supported by the National Natural Science Foundation of China, Provincial Natural Science Foundation of Guangdong, an IRRI Research Scholarship (to B. Liu), and grants from the Rockefeller 
Foundation and USAID (to H. Leung) and the Kansas State University Agricultural Experiment Station (to J. Leach). We thank many colleagues for providing the gene probes, $\mathrm{C}$. Vera Cruz and J. Wu for helpful discussion, and S. Hulbert and R. Nelson for reviewing the manuscript.

\section{LITERATURE CITED}

Boer, A. H. 2002. Plant 14-3-3 proteins assist ion channels and pumps. Biochem. Soc. Trans. 30:416-421.

Bonman, J. M., Khush, G. S., and Nelson, R. J. 1992. Breeding rice for resistance to pests. Annu. Rev. Phytopathol. 30:507-528.

Causse, M. A., Fulton, T. M., Cho, Y. G., Ahn, S. N., Chunwongse, J., Wu, K., Xiao, J., Yu, Z., Ronald, P. C., and Harrington, S. E. 1994. Saturated molecular map of rice genome based on an interspecific backcross population. Genetics 138:1251-1274.

Chen, D. H., Zeigler, R. S., Leung, H., and Nelson, R. J. 1995. Population structure of Pyricularia grisea at two screening sites in the Philippines. Phytopathology 85:1011-1020.

Close, T. J. 1996. Dehydrins: emergence of a biochemical role of a family of plant dehydration proteins. Physiol. Plant 97:795-803.

Cooper, B., Clarke, J. D., Budworth, P., Kreps, J., Hutchison, D., Park, S. Guimil, S., Dunn, M., Luginbuhl, P., Ellero, C., Goff, S. A., and Glazebrook, J. 2003. A network of rice genes associated with stress response and seed development. Proc. Natl. Acad. Sci. U.S.A. 100:4945-4950.

Druka, A., Kydrna, D., Kannangara, C. G., von Wettstein, D., and Kleinhofs, A. 2002. Physical and genetic mapping of barley (Hordeum vulgare) germin-like cDNAs. Proc. Natl. Acad. Sci. U.S.A. 99:850-855.

Faris, J. D., Li, W. L., Liu, D. J., Chen, P. D., and Gill, B. S. 1999. Candidate gene analysis of quantitative disease resistance in wheat. Theor Appl. Genet. 98:219-225.

Galvez, A. F., Gulick, P. J., and Dvorak, J. 1993. Characterization of the early stages of genetic salt-stress responses in salt-tolerant Lophopyrum elongatum, salt-sensitive wheat, and their amphiploid. Plant Physiol. 103:257-265.

Grant, J. J., and Loake, G. J. 2000. Role of reactive oxygen intermediates and cognate redox signaling in disease resistance. Plant Physiol. 124:21-29.

Hu, X., Bidney, D. L., Yalpani, N., Duvick, J. P., Crasta, O., Folkerts, O., and $\mathrm{Lu}, \mathrm{G} .2003$. Overexpression of a gene encoding hydrogen peroxide-generating oxalate oxidase evokes defense responses in sunflower. Plant Physiol. 133:170-181.

Jach, G., Gornhardt, B., Mundy, J., Logemann, J., Pinsdorf, E., Leach, R., Schell, J., and Maas, C. 1995. Enhanced quantitative resistance against fungal disease by combinatorial expression of different barley antifungal proteins in transgenic tobacco. Plant J. 8:97-109.

Lamb, C., and Dixon, R. A. 1997. The oxidative burst in plant disease resistance. Annu. Rev. Plant Physiol. Plant Mol. Biol. 48:251-275.

Levine, A., Pennell, R. I., Alvarez, M. E., Palmer, R., and Lamb, C. 1996. Calcium-mediated apoptosis in a plant hypersensitive disease resistance response. Curr. Biol. 6:427-437.

Liang, H., Maynard, C., Allen, R., and Powell W. 2001. Increased Septoria musiva resistance in transgenic hybrid poplar leaves expressing a wheat oxalate oxidase gene. Plant Mol. Biol. 45:619-629.

Lincoln, S. E., Daly, M. J., and Lander, E. S. 1993. Mapmaker/Exp. 3.0 and Mapmaker/QTL 1.1. Whitehead Institute, Cambridge, MA.

Michelmore, R. 2003. The impact zone: genomics and breeding for durable disease resistance. Curr. Opin. Plant Biol. 6:397-404.

Nishizawa, Y., Nishio, Z., Nakazono, K., Soma, M., Nakajima, E., Ugaki, M., and Hibi, T. 1999. Enhanced resistance to blast (Magnaporthe grisea) in transgenic Japonica rice by constitutive expression of rice chitinase. Theor. Appl. Genet. 99:383-390.

Notteghem, J. L., Chatel, M., and Dechanet, R.1981 Diallel analyze of two characteristics of rice resistance to Pyricularia oryzae. Page 301-
318 in: Comptes-rendus du symposium sur la resistance du riz a la pyriculariose. IRAT-GERDAT, Montpellier, France.

Paterson, A. H., Damon, A., Hewitt, J. D., Zamir, D., Rabinowitch, H. D., Lincoln, S. E., Lander, E. S., and Tanksley, S. D. 1988. Resolution of quantitative traits into Mendelian factors by using a computer linkage map of restriction fragment length polymorphisms. Nature 335:721726.

Paterson, A. H., Damon, S., Hewitt, J. D., Zamir, D., Rabinowitch, H. D. Lincoln, S. E., Lander, E. S., and Tanksley S.D. 1991. Mendelian factors underlyiing quantitative traits in tomato: comparison across species, generation and environments. Genetics 127:181-197.

Price, A., Knight, M., Knight, H., Cuin, T., Tomos, D., and Ashenden, T. 2002. Cytosolic calcium and oxidative plant stress. Biochem. Soc. Trans. 24:479-483.

Ramalingam, J., Vera Cruz, C. M., Kukreja, K., Chittoor, J. M., Wu, J. L., Lee, S. W., Baraoidan, M. R., George, M. L., Cohen, M., Hulbert, S., Leach, J. E., and Leung, H. 2003. Candidate resistance genes from rice, barley, and maize and their association with qualitative and quantitative resistance in rice. Mol. Plant-Microbe Interact. 16:14-24.

Rohlf, F. J. 1993. NTSYS-pc Numerical Taxonomy and Multivariate Analysis System. Exeter Publishing Co., Setauket, NY.

SAS Institute. 1989. SAS/STAT User's Guide, Version 6.4. SAS Institute, Inc., Raleigh, NC, U.S.A.

Schweizer, P., Pokorny, J., Abderhalden, O., and Dudler, R. 2000. A transient assay system for the functional assessment of defense-related genes in wheat. Mol. Plant-Microbe Interact. 12:647-654.

Sehnke, P. C., DeLille, J. M., and Ferl, R. J. 2002. Consummating signal transduction: the role of 14-3-3 proteins in the completion of signalinduced transitions in protein activity. Plant Cell 14(Suppl.):S339S354.

Shah, D. M. 1997. Genetic engineering for fungal and bacterial diseases. Curr. Opin. Biotechnol. 8:208-214.

Stuber, C. W., Lincoln, S. E., Wolff, D. W., Helentjaris, T., and Lander, E. S. 1992. Identification of genetic factors contributing to heterosis in a hybrid from two elite maize inbred lines using molecular markers. Genetics 132:823-839.

Temnykh, S., Park, W. D., Ayres, N., Cartinhour, S., Hauck, N., Lipovich, L., Cho, Y. G., Ishli, T., and McCouch, S. R. 2000. Mapping and genome organization of microsatellite sequences in rice (Oryza sativa L.) Theor. Appl. Genet. 100:697-712.

Vallelian-Bindschedler, L., Mosinger, E., Metraux, J. P., and Schweizer, P. 1998. Structure, expression and localization of a germin-like protein in barley (Hordeum vulgare L.) that is insolubilized in stressed leaves. Plant Mol. Biol. 37:297-308.

Wang, G., Mackill, D. J., Bonman, J. M., McCouch, S. R., and Nelson, R. J. 1994. RFLP mapping of genes conferring complete and partial resistance to blast resistance in a durably resistant rice cultivar. Genetics 136:1421-1434.

Ward, E. R., Uknes, S. J., Williams, S. C., Dincher, S. S., Wiederhold, D. L., Alexander, D. C., Ahl-Goy, P., Metraux, J. P., and Ryals, J. A. 1991. Coordinate gene activity in response to agents that induce systemic acquired resistance. Plant Cell 3:1085-1094.

Yap, I. V., and Nelson, R. J. 1996. WinBoot: a program for performing bootstrap analysis of binary data to determine the confidence limits of UPGMA-based dendrograms. Int. Rice Res. Inst. Discuss. Pap. Ser. 14

Young, N. D. 1996. QTL mapping and quantitative disease resistance in plants. Annu. Rev. Phytopathol. 34:479-501.

\section{AUTHOR-RECOMMENDED INTERNET RESOURCES}

International Rice Genome Sequencing Project (IRGSP) physical map of rice: www.tigr.org/tdb/e $2 \mathrm{k} 1 / \mathrm{osa} 1$

Kansas State University defense gene collection: www.ksu.edu/ksudgc 